\title{
CONSECUTIVE BITES ON TWO PERSONS BY THE SAME COBRA: A CASE
}

\section{REPORT}

\author{
AMIN MR (1), MAMUN SMH (2), CHOWDHURY NH (3), RAHMAN M (4), GHOSE A \\ (5), AL HASAN A (6), FAIZ MA (7)
}

(1) Consultant of Medicine, Hathazari Upazilla Health Complex, Chittagong, Bangladesh; (2) Assistant Professor of Cardiology, Chittagong Medical College Hospital, Chittagong, Bangladesh; (3) Assistant Professor of Neuromedicine, Comilla Medical College, Comilla, Bangladesh; (4) Assistant Professor of Gastroenterology, Shahabuddin Medical College, Dhaka, Bangladesh; (5) Resident Medical Officer, Chittagong General Hospital, Chittagong, Bangladesh; (6) Consultant of Medicine, Meghna Health Complex, Comilla, Bangladesh; (7) Professor of Medicine, Dhaka Medical College, Dhaka, Bangladesh.

ABSTRACT: In tropical countries like Bangladesh, persons are bitten by snakes every day and a considerable number of patients die en route to the hospital. An event of consecutive neurotoxic bites on two men by a single snake was observed in the Snake Bite Study Clinic (SBSC) of the Chittagong Medical College Hospital $(\mathrm{CMCH})$. Two brothers, working in their semi-pucca restaurant, were successively bitten by the same cobra on their lower limbs. Within an hour, they were taken to the $\mathrm{CMCH}$. Few minutes after admission, both developed symptoms of neurotoxicity: ptosis, nasal voice, dysphagia, broken neck sign, etc. They received polyvalent antivenom (Haffkine Bio-Pharmaceuticals Company, India) and other auxiliary treatment immediately. Within few hours, neurotoxic features were completely absent. Later, the snake was captured in the restaurant kitchen and identified as monocellate cobra (Naja kauthia) by the SBSC. The elder brother developed significant antivenom reactions and both presented necrosis and ulceration at the bite sites. In these cases, immediate arrival to the hospital and early administration of antivenom resulted in successful recoveries.

KEY WORDS: consecutive bites, neurotoxicity, cobra, snakebite, antivenom.

CONFLICTS OF INTEREST: There is no conflict.

\section{CORRESPONDENCE TO:}

MOHAMMAD ROBED AMIN, Consultant of Medicine, Department of Medicine, Hathazari Upazilla Health Complex, Chittagong, Bangladesh, 28 R. C. Church Road, Patherghata, Chittagong, Bangladesh. Email: robedamin@yahoo.com. 


\section{INTRODUCTION}

Envenomation resulting from snakebites is an important public health problem in many tropical and subtropical countries. It comprises a regional burden in South Asia, where its high incidences are reported during agricultural activity, therefore WHO considered it an occupational health hazard (14). The estimated prevalence of snakebites in Bangladesh is 4.3 cases per 100,000 inhabitants, presenting a mortality rate of $20 \%$; the highest numbers are recorded in Chittagong and Barisal Divisions (7 per 100,000) (3). A hospital-based study in Chittagong and Cox's Bazar showed that primary health care services like Thana Health Complex (THC) are not utilized by persons from rural areas for the management of snakebites and that four THC, out of 16, showed no snakebite case in a year (2). Most deaths provoked by snakebites, in Bangladesh, occur in communities and on the way to the hospital (4, 5 ); where, besides the scarcity of antivenom, it is also withheld from victims due to the danger of reactions.

In our country, only Polyvalent Haffkine ${ }^{\circledR}$ anti-snake venom, (Haffkine BioPharmaceuticals Company, India) is available. Adequate doses of this antivenom in addition to other auxiliary treatment, like neostigmine and ventilatory support, are crucial for saving the victims (6). Few persons (<10\%) brought the snakes, dead or alive, to the hospital; but most victims did not see the snake and could not identify the animal. The snakebites were presumed to be from: cobras (Naja), green snakes (Trimeresurus), kraits (Bungarus), sea snakes, Russell's vipers and others. An epidemiological study from the Snake Bite Study Clinic (SBSC), in Chittagong Medical College Hospital $(\mathrm{CMCH})$, in Bangladesh, revealed that most bites were due to cobra. The monocellete cobra is the most common venomous snake of the country (7). After a cobra bite, the anti-snake venom treatment usually reverses neurotoxic features, but it cannot prevent local tissue necrosis. Victims of neurotoxic snakebites frequently develop respiratory failure and sometimes this symptons are successfully managed with artificial ventilation along with anti-venom (3). Successive bites by a single snake, a case reported in Bangladesh, in 1999, almost resulted in fatal envenomation on two boys (8). One year after this incident, a similar occurrence was registered in the SBSC of $\mathrm{CMCH}$, when two consecutive bites by a monocellete cobra were observed. The present study describes the latter event with few observations. 


\section{CASE REPORT}

In the late afternoon of the $12^{\text {th }}$ of July 2000 , two brothers who were working in their own restaurant - a semi-pucca house surrounded by a brick wall, about 3 to $4 \mathrm{~km}$ away from city area - were bitten by the same snake.

The elder man was the cashier while the younger one was the waiter. When the event occurred, four customers were having lunch in the restaurant. The elder brother initially felt something crossing his legs; then he immediately moved and saw a snake (Figure 1). During the movement he was bitten on his right foot. Immediately, he felt intense pain and left the restaurant building chasing the animal. The snake, in turn, promptly changed its course and quickly entered the restaurant again. Customers were instantaneously gripped by panic and jumped over tables.

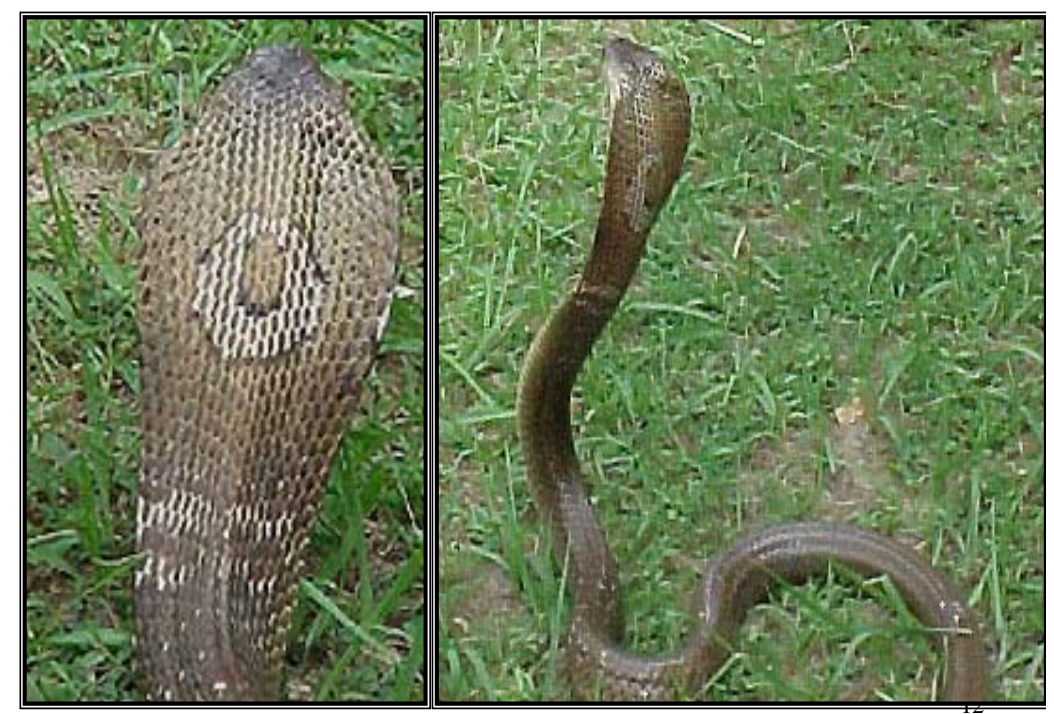

Figure 1. The involved cobra was observed and identified by the Snake Bite Study Group.

The elder man shouted at his younger brother to leave immediately. Despite the advice, the boy ran towards the animal that lift up its hoods, without delay, and bite him on the medial aspects of his left knee. After this, the snake rapidly moved towards the kitchen (Figure 2). The chef left the kitchen towards the dining area and asked for help. The two brothers were in deep aching pain and the elder one presented mild bleeding in his ankle. A customer and few volunteers immediately rescued both brothers. 


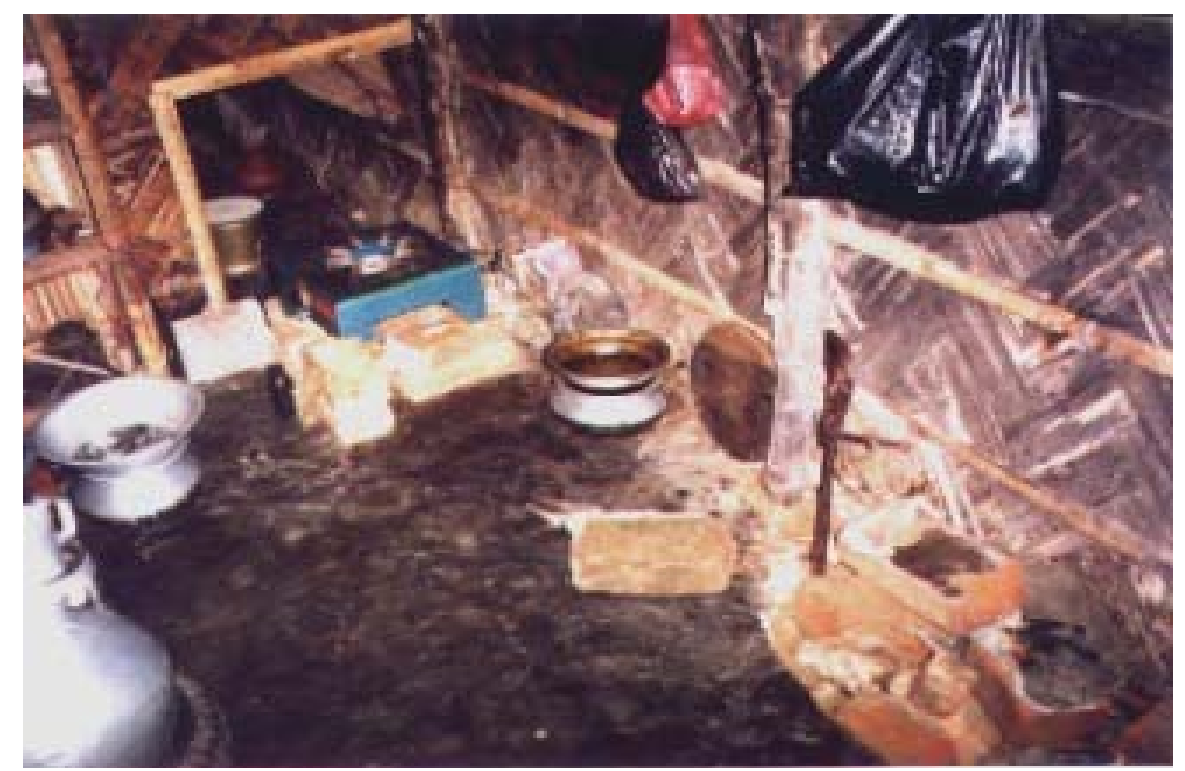

Figure 2. The kitchen where the cobra was captured by the chef.

Tight tourniquets were applied to the elder brother (case 1) by them with ropes and cloths. Peculiarly, they were giving more attention to the man; four tourniquets were applied to him and he was forthwith sent, accompanied by two attendants, to $\mathrm{CMCH}$ (Table1). These persons were convinced that snakes deliver venom only in the first bite, thus, in their judgment, the boy probably received no venom in the second attack. However, the boy (case 2) felt increasing pain, vomited 30 minutes after the incident and presented generalized weakness. One attendant, then, thought his symptoms were serious, applied three tight ropes above his knee and sent him to $\mathrm{CMCH}$ with three persons. In the meanwhile, the chef and few neighbors, carrying sticks, entered the kitchen to catch the animal. They found the snake near the oven, in a hole, captured it and sent the animal in a plastic bag to $\mathrm{CMCH}$. 
Table 1. Description of victim bites

\begin{tabular}{c|c|c}
\hline Characteristics & Case 1 & Case 2 \\
\hline Age & 37 years & 12 years \\
\hline Sex & Male & Male \\
\hline Locality & Inside the restaurant & Inside the restaurant \\
\hline Site of bite & Medial aspect of the right & $\begin{array}{c}\text { Medial aspect of the left } \\
\text { knee }\end{array}$ \\
\hline Time of bite & $12: 30$ p.m. & $12: 30$ p.m. \\
\hline Pre-hospital treatment & Four ligatures above knee & $\begin{array}{c}\text { Three ligatures above } \\
\text { knee }\end{array}$ \\
\hline Time between the bite and & & 1 hour and 25 minutes \\
\hline hospitalization & 45 minutes & \\
\hline
\end{tabular}

The physicians of the Snake Bite Study Clinic (SBSC), in $\mathrm{CMCH}$, received the victims in an interval of 40 minutes. They found classical fang marks in both patients that, additionally, did not show any neurological signs during admission. The two brothers were afflicted by intense pain and swelling (Table 2). The boy (case 2) was also vomiting, even after admission, and was very weak, while the elder brother (case 1) was drowsy. But none of them presented any sign of neurotoxicity during admission, in spite of the fact that they did not arrive simultaneously and the delayed first aid assistance to the second victim. 
Table 2. Patient clinical features during admission in SBSC

\begin{tabular}{c|c|c}
\hline Characteristics & Case 1 & Case 2 \\
\hline Local pain & Present & Present \\
\hline Bleeding from bite site & Present & Absent \\
\hline Pulse & $96 / \mathrm{min}$ & $108 / \mathrm{min}$ \\
\hline Blood pressure & $145 / 90 \mathrm{mmHg}$ & $100 / 60 \mathrm{mmHg}$ \\
\hline $\begin{array}{c}\text { Neurotoxic } \\
\text { manifestations }\end{array}$ & Drowsiness & None \\
\hline $\begin{array}{c}\text { Other features of } \\
\text { envenomation }\end{array}$ & None & None \\
\hline Fang marks & Two, $10 \mathrm{~mm}$ apart & Two, $10 \mathrm{~mm}$ apart \\
\hline
\end{tabular}

Thirty minutes after observation the elder brother (case 1) started developing ptosis followed by blurred vision, broken neck sign (Figure 3), dysphonia and dysphagia. All these classical neurological signs were also observed in the younger brother (case 2) about one and half hour after his admission (Table 3).

Table 3. Development of neurotoxic symptoms

\begin{tabular}{c|c|c}
\hline \multirow{2}{*}{ Signs of neurotoxicity } & Case 1 & Case 2 \\
\cline { 2 - 3 } & \multicolumn{2}{|c|}{ Time of onset after admission } \\
\hline Ptosis & 30 minutes & $1 \frac{1}{2}$ hours \\
\hline Blurred vision & 30 minutes & 1 hour \\
\hline Nasal voice & 1 hour & 2 hours \\
\hline Vomiting & Absent & $21 \frac{1}{2}$ hours \\
\hline Generalized weakness & 1 hour & 1 hours \\
\hline
\end{tabular}


Both brothers were submitted to total count (TC) and differential count (DC) of white blood cell (WBC) and platelet count. Routine urine examination, serum creatine phosphokinase (CPK), electrocardiogram (ECG) and peak expiratory flow rate (PEFR) were also performed. The two patients received neostigmine (subcutaneously, $50 \mu \mathrm{g} / \mathrm{kg} / 4$ hours), atropine (intravenously, $15 \mu \mathrm{g} / \mathrm{kg} / 4$ hours), polyvalent antivenom (Haffkine Bio-Pharmaceuticals Company, India) and antibiotics. Polyvalent antivenom dose administered was 100 cc (10 vials) according to WHO guidelines for snakebite management in Southeast Asia (SEARO guidelines). Each vial contains $10 \mathrm{cc}$ of lyophilized antivenom; thus, 10 vials correspond to $100 \mathrm{cc}$, which is the dose required to manage a single victim. Intravenous infusion is composed of reconstituted freeze-dried antivenom diluted in approximately 5 to 10 $\mathrm{mL}$ of isotonic fluid/kg of body weight (i.e. 250 to $500 \mathrm{~mL}$ of isotonic saline). The drug was infused at a constant rate for one hour. Both brothers were closely monitored for at least one hour after starting antivenom administration, with standby preparation of adrenaline, hydrocortisone, chlorpheniramine and ranitidine.

The elder brother (case 1) developed anaphylactic reaction 20 minutes after antivenom administration, presenting vomiting, urticaria, cough and respiratory difficulty (Figure 3). Antivenom was temporarily interrupted and anaphylaxis was managed with standard protocol. He recovered from anaphylactoid reaction and after a brief period of 10 minutes, the antivenom infusion was restarted. However, 5 minutes later, the patient developed urticaria, cough and abdominal pain once again. Antivenom was discontinued yet again and two doses of adrenaline were repeated within a 5 minutes interval. The patient recovered for a second time from the reaction with persistent neurotoxic manifestations. The physician, after consulting an expertise of SBSC, restarted the antivenom 10 minutes later. No more anaphylactic responses were recorded the patient. The antivenom was completed one hour after schedule and strangely the patient developed pyrogenic reaction thereafter, with chills, shivering, goosebumps and fever. These effects were easily managed with paracetamol suppository. Afterward, the patient recovered from the clinical signs of envenomation within approximately three and a half hours after arrival. No subsequent neurological deficit was seen (Table 4).

The younger brother (case 2), in the meanwhile, was treated with same dose of antivenom (100 cc) diluted in normal saline. Neostigmine and atropine were also given according to the protocol. He developed mild pyrogenic reaction only. 
Thereafter, he completely recovered from all neurological and systemic signs within two hours. The brothers were monitored over the next five days, both developed local envenomation in the forms of ulceration, blistering and necrosis (Figure 3). Expertise surgical care was undertaken and both patient stayed for three weeks in the hospital up to their complete recovery.

Table 4. Treatment in both cases

\begin{tabular}{c|c|c}
\multirow{2}{*}{ Treatment } & Case 1 & Case 2 \\
\cline { 2 - 3 } & \multicolumn{2}{|c}{ Number of doses } \\
\hline \hline Anti-snake venom & $\begin{array}{c}\text { Single dose (10 vials), } \\
\text { with interruption, given } \\
\text { in 3 hours }\end{array}$ & $\begin{array}{c}\text { Single dose (10 vials) in } \\
\text { one hour }\end{array}$ \\
\hline Neostigmine injection & 1 dose & 1 dose \\
\hline Atropine injection & 1 dose & 1 dose \\
\hline \hline $\begin{array}{c}\text { Adrenaline, } \\
\text { hydrocortisone and } \\
\text { antihistamine injections }\end{array}$ & 3 doses for anaphylaxis & Nil \\
\hline Paracetamol \\
suppository
\end{tabular}



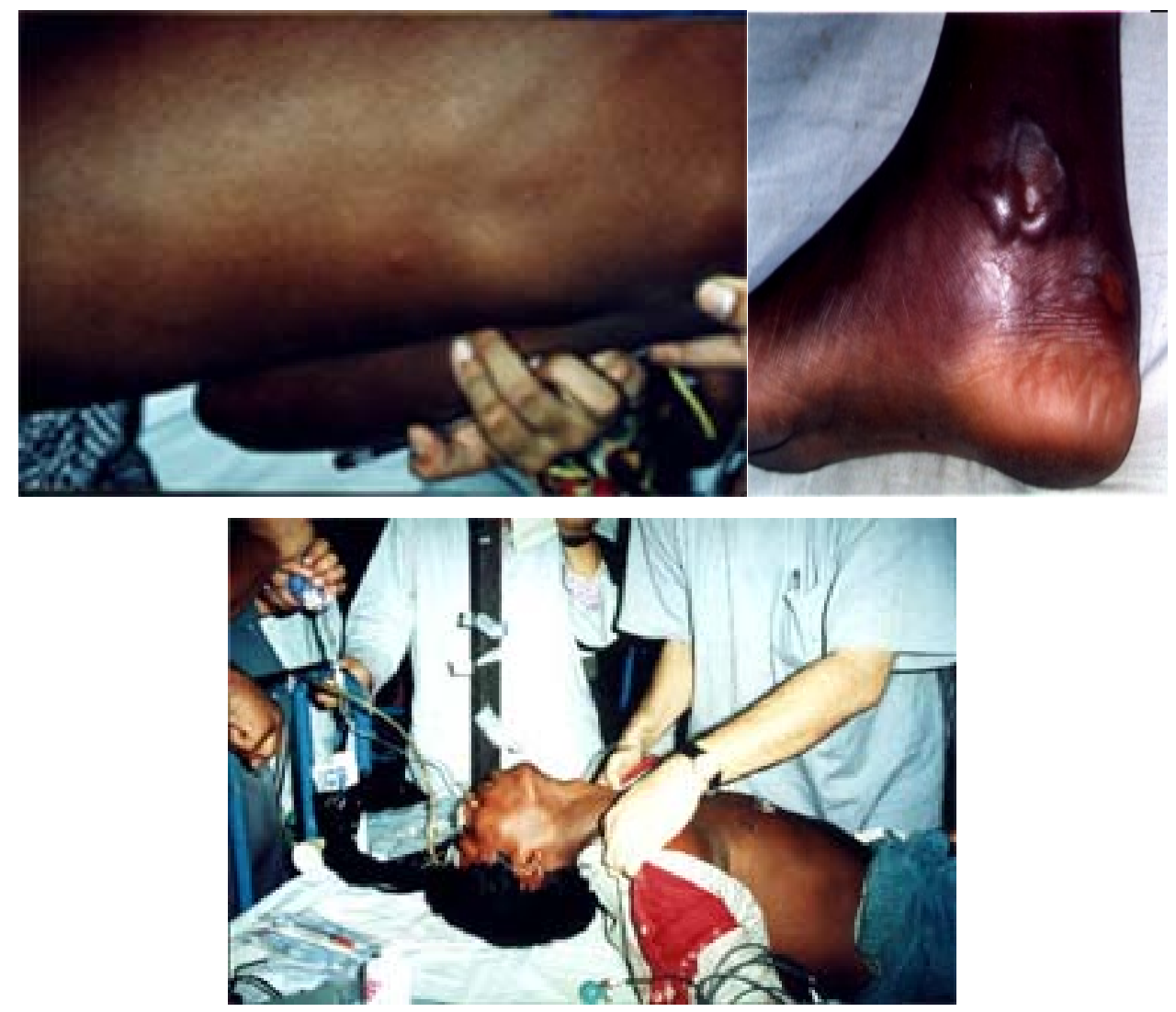

Figure 3. Urticaria, local blister and broken neck sign in the elder brother.

During the treatment, the Snake Bite Study Clinic identified the involved animal as a monocellate cobra, Naja kauthia, which was killed by the restaurant attendants. A professional from the Snake Bite Study Clinic visited the place where the bites had occurred and observed that it was a slum area surrounded by bushes, an environment suitable for snakes.

\section{DISCUSSION}

Successive venomous bites on two boys by a single snake, resulting in almost fatal envenomation, were first reported in Bangladesh, by the SBSC, in $\mathrm{CMCH}$, on 1999 (8). A similar event, reported in the current study, which involved two brothers, was also registered in the same hospital. The latter accident happened during the day, differently from the first report, in which the bites occurred at home while the two boys were sleeping. It has been reported that cobra strikes are more ineffective during the day; on the other hand, they are more dangerous at night, when snake eyesight is more acute (9). 
Cobras are not aggressive snakes and tend to escape when encountered in the wild. They strike only when accidentally touched or under strong provocation; when cornered, they spread their hoods, hiss, sway the body from side to side and strike repeatedly $(9,15)$. In the current report, it is possible to observe that the first bite was due to an accidental interaction, whereas the second one occurred because the snake felt threatened.

In view of the high proportion of bites, by some venomous species that results in little or no envenomation, it was suggested that snakes may be capable of biting defensively without injecting venom. Some viperidae species can inject up to ten lethal doses of venom (to their natural prey) in consecutive strikes $(9,10)$. The quantity of venom injected in each strike appears to be very variable and the popular belief that snakes are less dangerous after feed is not true $(9,10,15)$. These reptiles do not exhaust their store of venom, even after several strikes, and do not become less venomous after eating their prey $(10,15)$.

Envenomation by Naja naja, Naja kauthia and Ophiophagus hannah (king cobra) causes almost immediate severe local pain and blister formation, as well as lymphadenitis may occur (9). Cobras are capable of producing neurotoxic symptoms in human victims. Early symptoms, before the occurrence of the neurological signs, include vomiting, eyelid heaviness, blurred vision, drowsiness and, sometimes, paraesthesia of the mouth, tongue, lips, headache and dizziness (13). Paralysis started by ptosis and external ophthalmoplegia, which may be evident minutes after the bite, although it is more often after a delay of many hours $(9,10)$. Neurotoxicity progress may involve the palate, jaws, tongue, vocal cords, neck and deglutition muscles (1). Respiratory failure may be precipitated by airway obstruction at this stage, or later after paralysis of intercostals muscles and diaphragm.

In the present study, the early drowsiness of the elder brother and the repeated vomiting of the younger one could be considered signs of lethal bites. These nonspecific findings were observed in the SBSC, where they were followed by classical neurological findings. Neurotoxic effects are completely reversible, either acute response to antivenom or after spontaneous resolution over several days or weeks (14). Neurotoxic symptoms of envenomation by cobra (postsynaptic toxins) may begin to improve 30 minutes after antivenom administration, but usually it takes several hours (12). Presynaptic toxins (from kraits and sea snakes) are unlikely to 
respond in this way (12). In our study, the response to antivenom was highly satisfactory and anticholinesterase also seemed very effective.

Although the elder brother had interrupted antivenom infusion, due to repeated anaphylactic responses, all his neurological features completely disappear after the treatment, which suggests its efficacy. Both brothers received only one dose of antivenom, which also indicates its effectiveness and neutralizing capacity against cobra toxins. Early anaphylactic reactions usually manifest within 10 to 20 minutes after starting intravenous antivenom injection, or within 30 to 180 minutes of starting intravenous infusion of diluted antivenom. Premonitory symptoms include restlessness, cough, scalp itching, nausea, vomiting, a feeling of heat and palpitation. Later, diffuse urticaria, generalized pruritus, fever, tachycardia and autonomic manifestation can also appear. In more than $40 \%$ of the reactions, potentially fatal hypotension, airflow obstruction, bronchospasm, laryngospasm, and angioedema are also recorded. However, fatal reactions are rare.

The incidence of reactions varies from 3 to $54 \%$ of the cases; it usually decreases with smaller doses and refined antivenoms, and it is generally greater when the intravenous route, rather than the intramuscular one, is used (12).

In the current study, the polyvalent antivenom was employed causing pyrogenic reactions, in both brothers, and repeated anaphylactic features, in the elder one, which indicates the danger of polyvalent antivenoms. It also reveals the need of monospecific antivenoms, which are highly effective and do not cause adverse reactions. At the earliest sign of anaphylaxis, epinephrine (adrenaline) should be administered (intramuscularly) in an initial dose of $500 \mu \mathrm{g}(0.5 \mathrm{mg})$ for adults, 0.01 $\mathrm{mg} / \mathrm{kg}$ of body weight for children. Severe, life-threatening anaphylaxis can evolve very rapidly and consequently epinephrine (adrenaline) must be given at the very first sign of a reaction, even when a few spots of urticaria have appeared or at the start of itching, tachycardia or restlessness. The dose can be repeated every 5 to 10 minutes if the patient condition is deteriorating (11). In this report, the elder brother developed an anaphylactic response, after which he was quickly submitted to treatment and presented, at last, complete recovery. The effectiveness of adrenaline in early reaction suggests a need for prophylactic adrenaline before the use of anti-snake venom, especially polyvalent ones. 


\section{CONCLUSIONS}

The current report is the second occurrence of consecutive bites by the same snake in Bangladesh, which indicates that cobras are the most commom venomous snakes in the country and that Naja kauthia (monocellate cobra) may be the most frequent species among them. The envenomation signs presented by the brothers reveal that cobra venom is very effective in inducing local tissue necrosis.

Prompt assessment, observation and early specific management are the keys to treat severe envenomations. Anti-snake venoms, preferable monovalent ones, are highly effective in addition to auxiliary treatment like anticholinesterase and respiratory support. Reactions to polyvalent antivenom are not uncommon and may lead to lethal anaphylaxis, but prompt action with adrenaline can easily control the situation. Guidelines must be followed by physicians for management of snakebites in Bangladesh.

\section{ETHICAL APPROVAL}

The present study was approved by the Ethical Review Committee of the $\mathrm{CMCH}$. All patients gave written or verbal consent.

\section{REFERENCES}

1 CHIPPAUX JP., WILLIAMS V., WHITE J. Snake venom variability: methods of study, results and interpretation. Toxicon, 1991, 29, 1279-303.

2 FAIZ MA., CHOWDHURY SK., HUSSAIN I. Snake bite in Chittagong and Cox'S Bazar: a hospital based study. Bangladesh J. Med., 1997, 8, 52-7.

3 FAIZ MA., RAHMAN MR., DAS KK. Non-poisonous snake bite in Chittagong Medical College Hospital, Chittagong. Bangladesh Med. J., 1999, 28, 3-5.

4 FAIZ MA., RAHMAN MR., YOUNUS EB. A hospital based study of snake bite in Chittagong Medical College. J. Bang. Coll. Phys. Surg., 1995, 13, 3-8.

5 FAIZ MA., RASHID R., GAFUR MA. Observation of 10 fatal cases following snakebite in Chittagong, 1993-1998. Bangladesh J. Med., 1999, 10, 30-3.

6 FAIZ MA., RIDWANUR R., YUNUS EB., AMIR MH., FAZLE ECEY., MARGUB M., DUTTA MK., BEGUM Z. Snake bite: is it a public health problem in Bangladesh? Bangladesh J. Med., 1995, 23, 61-4 
7 HUQ F., ISLAM MA., SARKER MH., CHOWDHURY AHMW., KABIR MM. Epidemiology of snakes in Bangladesh. Bangladesh J. Zool., 1995, 23, 61-4.

8 MAMUN SMH., FAIZ MA., RAHMAN R., CHOWDHURY FE., WAHAB MA., ATAHER QS. Two consecutive venomous bites by the same snake: a case report. Society of Medicine $1^{\text {st }}$ Conference, 2000.

9 MEIER J., WHITE JI. Clinical toxicology of animal venoms and poisons. Boca Raton, Florida: CRC Press, 1995. 553-6p.

10 MINTON SA. Neurotoxic snake envenoming. Semin. Neurol., 1990, 10, 52-61.

11 SUTHERLAND SK. Antivenom use in Australia. Premedication, adverse reactions and the use of venom detection kits. Med. J. Aust., 1992, 157, 734-9.

12 THEAKSTON RD., PHILLIPS RE., WARRELL DA., GALAGEDERA Y., ABEYSEKERA DT., DISSANAYAKA P., DE SILVA A., ALOYSIUS DJ. Envenoming by the common krait (Bungarus caeruleus) and Sri Lankan cobra (Naja naja naja): efficacy and complications of therapy with Haffkine antivenom. Trans. R. Soc. Trop. Med. Hyg., 1990 , 84, 301-8

13 WARRELL, DA. Clinical toxicology of snake bite in Asia. In: MEIER, J., WHITE J. (eds.) Handbook of clinical toxicology of animal venoms and poisons. Boca Raton, Florida: CRC Press, 1995. 493-594p.

14 WARRELL DA. Snake venoms in science and clinical medicine. 1. Russell's viper: biology, venom and treatment of bites. Trans. R. Soc. Trop. Med. Hyg., 1989, 83, 732-40.

15 WUSTER W., THORPE RS. Asiatic cobras: systematics and snakebite. Cell. Mol. Life Sci., 1991, 47, 205-9. 